\title{
Hinweise für den Benutzer
}

Gliederung

I. Zur Stichwortauswahl . . . . . . . . . . . . . . . . . VIII

II. Zum Stichwortansatz . . . . . . . . . . . . . . . . . . . . . . . . IX

III. Zur Definition der Lexeme . . . . . . . . . . . . . . . . . . . . . . XI

IV. Zum Aufbau der Wörterbuchartikel . . . . . . . . . . . . . . . . . . XIV

V. Zu den grammatischen Angaben . . . . . . . . . . . . . . . XVI

VI. Zur Darstellung des Kontextes . . . . . . . . . . . . . . . . . . . . XXI

VII. Zu den stilistischen Kennzeichnungen und Anwendungsbedingungen . . . . XXII

VIII. Zu den Akzent- und Ausspracheangaben . . . . . . . . . . . . . . . . XXIV

IX. Typographisches . . . . . . . . . . . . . . . . . . . XXVII

X. Abkürzungsverzeichnis .. . . . . . . . . . . . . . . . XXIX

\section{Zur Stichwortauswahl}

Das Handwörterbuch erfaßt vorrangig den Teil der Lexik, 'den man zum Zentrum der Allgemeinsprache rechnen darf, der der nichtfachgebundenen Kommunikation dient, weitgehend gruppenunspezifisch ist und weitgehend überregional verwendet wird. Im Gegensatz dazu und außerhalb des Zentrums, an der Peripherie, stehen die Fachwortschätze und diejenigen landschaftlich gebundenen Lexeme (Regionalismen), die in der Allgemeinsprache nicht häufig vorkommen. Die Grenzen zwischen Zentrum und Peripherie sind jedoch fließend. In dem Maße, wie im Alltag Fachgebiete jeweils in das Zentrum des allgemeinen Interesses rücken, wechseln Fachtermini in die Randzone der Allgemeinsprache über. So haben Fachgebiete wie Wirtschaft, Sport, Technik u. a. die Allgemeinsprache wesentlich bereichert.

Der Anteil der einzelnen Fachwortschätze an der Lexik der Allgemeinsprache ist recht unterschiedlich. Dem trägt das Handwörterbuch Rechnung, indem es bei der Stichwortauswahl besonders die Fachgebiete berücksichtigte, die in unserem Alltag eine besondere Rolle spielen.

Bei der Stichwortauswahl wurde der Wortschatz der letzten dreißig Jahre zugrunde gelegt. Damit ist die Aufnahme veralteter Lexeme ausgeschlossen (z. B. Sozietät, Siechenhaus). Aufgenommen wurden lediglich die Lexeme mit der Tendenz zum Veralten (z. B. wohlerzogen). Historische Lexeme, d. h. Lexeme, die Erscheinungen vergangener Epochen widerspiegeln, wurden unter dem Gesichtspunkt ihrer Gewichtigkeit ausgewählt (z. B. Sklave). Die Auswahl ideologierelevanter Stichwörter folgt den allgemeinen Prinzipien der Stichwortauswahl, doch finden in diesem Bereich bestimmte Lexeme Berücksichtigung, die nach 1945 erst neu entstanden und heute bereits veraltet sind. Es handelt sich dabei um Lexeme, die bestimmte Etappen der jüngeren historischen Entwicklung widerspiegeln und für die Darstellung dieser Zeit weiterhin Verwendung finden. Es sind dies Wörter wie Neubauer, Neulehrer, Arbeiter- und -Bauern- 
Fakultät. Bei der Auswahl des regionalen Wortschatzes hatten die landschaftlich gebundenen Lexeme, der DDR und der BRD den Vorrang, aber auch die in Österreich und die im deutschsprachigen Teil der Schweiz gebräuchlichen sprachlichen Besonderheiten wurden in gewissem Umfang berücksichtigt.

Der Aspekt Zentrum-Peripherie wurde nicht ausschließlich auf die Auswahl der zeitlich und landschaftlich eingeschränkten Lexeme sowie der terminologischen Begriffe bezogen, sondern auch auf den morphologisch-semantischen Bereich: Bei der Auswahl blieben Komposita und Ableitungen (mit reihenbildendem Charakter), deren Bedeutung durch die Summe ihrer Bestandteile transparent wird, weitgehend unberücksichtigt (z. B. die Feminina auf -in; aufgenommen wurden nur sehr übliche Formen, wie z. B. Verkäuferin, Lehrerin; auf Reiterin o. ä. wurde verzichtet).

Abkürzungen wie Abs. für Absender wurden nicht als selbständige Stichwörter aufgenommen, sondern unter ihrer Vollform aufgeführt. Als selbständige Stichwörter erscheinen dagegen in einer Auswahl Kurzwörter und Initialwörter (z. B. LPG, FKK, Akku, Uni). Diminutivformen wurden nur dann berücksichtigt, wenn sie in ihrer Bedeutung von ihrem Basislexem abweichen (z. B. Dämchen, Pärchen, Mütehen). Eigennamen fanden nur dann Berücksichtigung, wenn sie als Appellativa oder als Bestandteile von Phraseologismen (s. II.8) verwendet werden (z. B. spanisch: spanische Wand, Frankreich: leben wie Gott in Frankreich).

\section{Zum Stichwortansatz}

1. Unter einem Stichwort verstehen wir das Wort, das am Beginn des Wörterbuchartikels steht. Es ist halbfett gedruckt. Seine Rechtschreibung richtet sich nach der 17., neubearbeiteten Auflage des Duden. Wörter mit Bindestrich (fifty-fifty) oder mehrgliedrige Ausdrücke ohne Bindestrich (ad infinitum) werden als ein Stichwort behandelt. Orthographische, grammatische oder regionale Varianten (Friede, Frieden; placieren, plazieren) werden auf die heute übliche Form oder die hochsprachliche Form verwiesen.

2. Die Stichwörter sind streng alphabetisch geordnet. Bei gleichlautenden, in der Groß-, Kleinschreibung voneinander abweichenden Stichwörtern rangiert Kleinschreibung vor Großschreibung (pfropfen, Pfropfen). Stichwörter mit Umlaut rangieren hinter Stichwörtern ohne Umlaut (nutzen, nützen). Stichwörter, die verschiedenen Wortarten zugeordnet werden (1'auf /Präp./, ${ }^{2}$ auf /Adv./) oder sich durch ihr Genus voneinander unterscheiden ('Band, das, ${ }^{2}$ Band, 'der, ${ }^{3} B a n d$, die [bent] oder hinsichtlich ihrer Flexion. divergieren ('hängen ..., ${ }^{2}$ hängen /sw. $V b$./), werden in der Regel durch Indizes (vorangestellte Hochzahlen) gegliedert. Substantivierte Lexeme werden nur dann als selbständige Stichwörter angesetzt, wenn sie semantisch modifiziert und eigenständig sind (hoch, das Hoch = Meteor.). Substantivierungen ohne Bedeutungsänderung gegenüber ihrer Basis werden, soweit sie eine gewisse Verbreitung aufweisen, unter dem klein geschriebenen Stichwort mit abgehandelt.

3. Kurzwörter und Initialwörter werden in einer begrenzten Auswahl als selbständige Stichwörter behandelt, wenn sie gegenüber der Vollform hinsichtlich ihrer Verbreitung überwiegen (H0) oder gleichberechtigt sind (LPG, LKW).

4. Diminutivformen erscheinen nur dann als selbständige Stichwörter, wenn sie semantisch oder stilistisch von ihrer Basis abweichen (vgl. I). 